\title{
La cobertura de la burbuja inmobiliaria en la prensa española (2003-2013). Un análisis de las opiniones de las fuentes políticas y expertos
}

\section{The Housing Bubble in the Spanish Press (2003-2013). An analysis of the opinions of political and expert sources}

\author{
Arrese, A., Vara, A., Salvatierra, S. M. y Álvarez, J. L. 1 \\ Recibido: 28-05-2018 - Aceptado: 10-07-2018 \\ DOI: https://doi.org/10.26441/RC17.2-2018-A2
}

RESUMEN: Este estudio analiza las opiniones de políticos y expertos publicadas en cinco diarios españoles (los tres principales periódicos nacionales -El País, El Mundo y $A B C$-, y los dos principales periódicos financieros -Expansión y Cinco Días-) antes y después de la explosión de la burbuja inmobiliaria española. La metodología básica del estudio es el análisis de contenido, cuantitativo y cualitativo, de informaciones publicadas en los diarios citados entre 2003 y 2013 . El trabajo se centra en el análisis estadístico de coincidencias y divergencias en las declaraciones y opiniones de esas fuentes en torno a la naturaleza, causas y consecuencias de la burbuja. La hipótesis general de la investigación es que antes de la crisis, especialmente entre los políticos, no hubo un verdadero debate sobre la amenaza de una burbuja del sector inmobiliario, ni sobre sus posibles causas y consecuencias probables, con lo que dominó en la opinión autorizada un consenso "negacionista".

Palabras clave: cobertura periodística; burbuja inmobiliaria; periodismo económico.

ABSTRACT: This study compiles and analyzes data on views of politicians and economists reported in news articles published in five Spanish dailies (the three leading national newspapers -El País, El Mundo and $A B C$-, and the two leading financial newspapers -Expansión and Cinco Días-). Quantitative content analysis will be used as the basic methodology for research. This content analysis will be focused on the coincidences and divergences between the nature, structure and frames of the opinions and comments on the bubble made by these policy makers and experts in two different periods, before and after the bubble burst. The general hypothesis of the research is that before the crisis -especially among politicians- there was not a real discussion on the threat of a housing bubble, its causes and probable consequences, but a quite orchestrated "negationist" consensus.

Keywords: news coverage; housing bubble; economic journalism.

\footnotetext{
Ángel Arrese es Doctor en Comunicación y Profesor Titular de Periodismo en la Facultad de Comunicación de la Universidad de Navarra. Imparte Periodismo Económico, Marketing, Publicidad y Relaciones Públicas. aarrese@unav.es, https://orcid.org/0000-0002-0586-4188

Alfonso Vara es Doctor en Comunicación por la Universidad de Navarra y profesor visitante de la School of Journalism de la University of North Carolina at Chapel Hill. Imparte Introducción a la Economía, Principios de Periodismo Económico, Empresa de Comunicación. avara@unav.es, http://orcid.org/0000-0003-4271-1858

Stella M. Salvatierra es Doctora en Estadística por la Carnegie Mellon University (Estados Unidos) y Profesora Contratada del Departamento de Economía en la Facultad de Comunicación de la Universidad de Navarra. ssalvat@unav.es

José Luis Álvarez es Doctor en Economía Aplicada y Profesor en la Facultad de Ciencias Económicas y Empresariales de la Universidad de Navarra donde imparte Principios de Microeconomía, Economía y Finanzas y Diálogos: agenda internacional. jlalvarez@unav.es, http://orcid.org/0000-0002-7431-5334
} 


\section{Introducción}

La crisis económica y financiera que comenzó en Estados Unidos y Europa en 2008 es un magnífico objeto de estudio para analizar el discurso de los medios en materia económica. De hecho, en los últimos años ha habido muchas investigaciones que han abordado distintas dimensiones de la cobertura mediática de la crisis, tanto desde perspectivas domésticas como en estudios comparados. Una de las ideas recurrentes que se deriva de esas investigaciones es que en los años previos a la crisis los medios periodísticos fueron incapaces de advertir y de alzar la voz de alarma sobre los riesgos existentes en el sector financiero, en el inmobiliario, etc. (Schiffrin, 2011; Usher, 2012; Starkman, 2014; Arrese y Vara, 2014; Kalogeropoulos et al., 2015; van Dalen et al., 2015; Mercille, 2016). Ya en medio de la crisis, los medios fueron a menudo criticados por su cobertura simplista y acrítica de la situación, que en muchos casos se tradujo finalmente en una visión excesivamente alarmista sobre los problemas económicos y financieros que se vivían en esos años (Tulloch, 2009; Arlt y Storz, 2010; Fahy et al., 2010; Uchitelle, 2011; Titley, 2012; Quiring y Weber, 2012; Arrese, 2015a; Knowles et al. 2017).

Sin embargo, a pesar de ese interés creciente por la relación entre los medios y su cobertura de la crisis económica y financiera, quedan muchos temas y aspectos por analizar en torno a manifestaciones particulares de esa época. En el caso de España, no cabe duda que existe un fenómeno econó- mico y financiero concreto que sobresale respecto a todos los demás a la hora de explicar nuestra particular crisis: la "burbuja inmobiliaria". Como señala García Montalvo (2009):

A estas alturas ya nadie duda de la importancia que el sector inmobiliario ha tenido en la detonación y la propagación de la crisis económica actual. El origen de los problemas actuales hay que buscarlo en la formación de una burbuja inmobiliaria en muchos países y, en particular, en España (p. 32).

Esta aceptación generalizada de la "burbuja inmobiliaria" como epicentro del especial terremoto económico y financiero español no es sólo una cuestión de expertos. Los ciudadanos y los políticos saben que el desastre inmobiliario explica en buena parte la crítica situación de nuestro desempleo, del precario estado de las finanzas públicas, y la fragilidad del sistema financiero.

Por este motivo, al menos en el caso español, quizá no haya mejor tema para valorar la actuación de los medios durante la crisis que el tratamiento que han dado a la "burbuja inmobiliaria". Este artículo trata de abordar algunos aspectos de esa actuación. Para ello, tomando como referencia la explosión de la burbuja en 2007, se analiza las posturas de periodistas, políticos y expertos sobre el fenómeno de la burbuja inmobiliaria -la principal causante de la crisis económica y financiera española-, tal y como fueron hechas públicas en los principales diarios generalistas y económicos del país. Para llevar a cabo este análisis, en primer lugar se contextualiza la investigación en el marco de los trabajos 
realizados sobre la relación entre los medios y las burbujas especulativas, y en particular en el caso de las burbujas inmobiliarias. En segundo lugar, se describen los rasgos fundamentales de la burbuja inmobiliaria española, cuyas características permiten establecer dos períodos claramente diferenciados de análisis, antes y después de su explosión, a mediados de 2007. Posteriormente, se plantean las preguntas de investigación del trabajo y la metodología de análisis de contenido utilizada. Por último, se exponen los resultados del estudio empírico y las conclusiones.

\section{Burbujas y medios}

El concepto de "burbuja inmobiliaria" no es un concepto neutro. Como tantos otros en el ámbito de la economía -“crisis", "estancamiento", "recesión”, "especulación”, etc.- está cargado de significados. Técnicamente, una burbuja, en materia económica, se produce cuando los precios de los activos exceden su valor fundamental porque sus actuales propietarios creen que pueden revenderlos a un precio incluso mayor en el futuro (Brunnermeir, 2008). De esta forma, la existencia de tal fenómeno -ya sea en el mercado de activos inmobiliarios o en otros mercados- se manifiesta en una rápida y continuada elevación de precios, no justificada suficientemente por razones fundamentales -aumento de renta, por ejemplo- sino por expectativas de incrementos futuros de precios. En la práctica, la cuestión fundamental consiste en determinar si en un mercado, en cierto espacio de tiempo, se está pro- duciendo o no un fenómeno de burbuja, pues si así fuera, las burbujas necesariamente explotan si no se ponen remedios a las causas que las generan.

Pero más allá de su significado técnico, el contenido simbólico del término está asociado, en sus aspectos negativos, a conceptos como "alto riesgo", "fragilidad", "incertidumbre", "inestabilidad". Todos ellos están relacionados con las nefastas consecuencias -históricamente bien conocidas- de las explosiones de burbujas, sobre todo financieras, que en cierta forma se pueden entender como las consecuencias del alto riesgo que se asume en procesos de enriquecimiento fácil, de especulación, etc. En este sentido, aceptar o reconocer abiertamente la existencia de una burbuja significa asumir que puede explotar, y por tanto implica crear una situación de alarma que requiere urgente intervención. Al mismo tiempo, el pánico, en esas circunstancias, puede desencadenar el desastre.

Teniendo en cuenta estas ideas, se entiende que la utilización en el discurso público de la expresión burbuja sea enormemente sensible, que en torno a ella se genere controversia, y que a menudo se evite su utilización. Pero cuando existen suficientes motivos para alertar a la población de la posible existencia de este fenómeno, es responsabilidad de los medios hacer públicas esas controversias, exponer los argumentos que justifican o no la existencia de la burbuja, analizar sus posibles causas y consecuencias.

De hecho, los medios son actores fundamentales en mercados donde las 
opiniones influyen significativamente en los precios, aquellos en los que se pueden generar burbujas, tales como las bolsas de valores $\mathrm{u}$ otros mercados de activos susceptibles de espirales especulativas. Hace ya más de un siglo, Ellis (1892) expresaba esta idea claramente al afirmar:

Quizá la fórmula más sencilla para explicar la situación de un mercado es decir que el mercado muestra siempre un equilibrio entre dos presiones en conflicto -la presión para vender y la presión para comprar-. La opinión es a menudo la causa inmediata de esas presiones, y por ello, tener en cuenta los estados de opinión es de máxima importancia para entender y debatir sobre la situación y la naturaleza de los mercados (p. 109).

Desde entonces, muchas investigaciones han analizado diferentes tipos de interacción entre los medios, la opinión y la evolución de los mercados (véase, para una síntesis histórica, Schuster, 2006). Recientemente, Tetlock (2007), por ejemplo, concluye que el pesimismo de los medios sobre los mercados bursátiles presiona a la baja los precios de las acciones, lo que sugiere que el tono de la información periodística influye en la opinión de los operadores. Otros estudios destacan la importancia de los medios en la provisión de información para los inversores que se fijan en los mercados de valores (Chan, 2003; Peress, 2014), o el interés de tener en cuenta los efectos de las noticias en ámbitos como el del gobierno corporativo (Dyck y Zingales, 2003), la rentabilidad de las inversiones (Fang y Peress, 2009), el movimiento de las acciones (Dougal et al., 2012; Engelberg y Parsons, 2011), las situaciones de pánico en el mercado (Kleinnijenhuis et al., 2013), o el comportamiento de compra de los inversores individuales e institucionales (Lee, 1992; Barber y Odean, 2008). Por supuesto, las nuevas tecnologías y la nueva lógica de funcionamiento electrónico y automatizado de muchos mercados -sobre todo los bursátiles- produce nuevos fenómenos de interacción entre las noticias y los precios (Strauss et al. 2016, 2017; Strauss, 2018).

Pero la influencia de los medios es especialmente relevante cuando los precios de los activos pueden generar burbujas. El análisis del papel de los medios de comunicación en torno a la emergencia de burbujas económicas y financieras tiene una cierta tradición en los estudios sobre economía y periodismo, y a menudo los estudios clásicos en este campo incluyen a los medios como actores importantes en esas situaciones (Kindleberger, 1978; Garber, 2000). Históricamente, desde las primeras burbujas financieras que se recuerdan, los investigadores y los analistas se han fijado en el modo en el que los medios -sobre todo los periódicos- han contribuido a hinchar las burbujas, a difundir entre los inversores informaciones que alimentan expectativas exorbitadas. Shiller (2000) ha comentado que "la historia de las burbujas especulativas comienza a grandes rasgos con el nacimiento de los periódicos" (p. 85). Desde las burbujas holandesas y británicas de 
los siglos XVII y XVIII (Garber, 1989; Dale, 2004) hasta las más recientes, como la burbuja bursátil de las punto. com (Kurtz, 2000; Augey and Brin, 2004), la mayoría de los estudios realizados llegan a la conclusión de que los medios son un agente fundamental en la fase de boom de la burbuja, que antecede a su explosión (Chancellor, 2000).

En términos generales, se ha aceptado la idea de que los medios no cumplen con la función de alertar sobre los riesgos derivados de la generación de situaciones de burbuja, y sobre las posibles consecuencias de su explosión. Más bien, al contrario, actúan de forma acrítica, como cheerleaders del espíritu especulativo del mercado (Kurtz, 2000; Theil, 2014), en lugar de equilibrar y atemperar su posición, dando voz a los prophets of doom, que siempre existen en estos casos. Shiller $(2000,2001,2008)$ y Shiller y Akerlof (2009) sugieren que por medio de las historias que se escriben, y el sentido de realidad que producen, o por medio del interés que despiertan las noticias, los medios son propagadores centrales de los movimientos especulativos de precios. En esta misma línea, J.K. Galbraith escribió en su $A$ Short History of Financial Euphoria (1990) que los periodistas, y algunos otros que hablan claro durante los tiempos de euforia colectiva sobre el crecimiento económico, pueden convertirse en la excepción ante el amplio consenso y la norma generalizada por la que los intereses personales, la presión pública y la supuestamente superior opinión del mundo de las finanzas conspiran para mantener la euforia del mercado (citado en Davis, 2010: 199).

Junto a esta visión genérica del rol de los medios, un tanto tópica, en los últimos años, al hilo de un análisis más detallado del papel de la información periodística en los mercados financieros, se han publicado algunos trabajos que relativizan el papel de la información pública en las épocas de boom y de crash. Campbell, Turner y Walker (2012), por ejemplo, han analizado con detalle su protagonismo en la Manía de los Ferrocarriles que tuvo lugar en Gran Bretaña a mediados de la década de 1840, concluyendo que:

aunque la cobertura de los medios siguió el ritmo de los acontecimientos, y jugó un rol importante en la diseminación de la información, no se puede afirmar que amplificara los movimientos de precios durante la fase de manía, ni tampoco que contribuyera a la posterior explosión de la burbuja de las compañías del sector (p. 461).

De forma parecida, en este caso tomando como referencia la burbuja de las punto-com a finales del siglo XX, Bhattacharya et al. (2009) señalan que el bombo mediático que se dio al sector de Internet no explica la subida especulativa del precio de las acciones de ese tipo de compañías. Los medios, según estos estudios, no parecen tener un protagonismo tan grande en la intensificación de la fase expansiva de la burbuja, aunque sin duda son un factor más, que ayuda a explicar la evolución de los mercados en ciertos momentos. Desafortunadamente, no existe investigación suficiente sobre la posibilidad de 
que los medios actuaran como factor de alerta o freno de los procesos de especulación que subyacen en las burbujas, más allá de algunas referencias a casos particulares de periodistas o publicaciones que en tales situaciones han podido ir contracorriente -caso del New York Times en la crisis de 1929 (Galbraith, 1955)-, enviando ciertas señales débiles que, en cualquier caso, no fueron suficientes para actuar de verdaderas alertas (Ojala and Uskali, 2007).

Prácticamente todos los estudios citados analizan la actuación de los medios en las fases de la burbuja previas a la explosión, tanto desde el punto de vista de las prácticas profesionales como del análisis de contenidos. La investigación, sin embargo, es casi inexistente por lo que se refiere a su actuación con posterioridad. Por lo general, el tipo de análisis que se realiza es el de asunción o no de responsabilidades una vez que el crash se ha producido, y la búsqueda de causas y de responsables por su deficiente actuación. "Why the media did not bark?" suele ser un enfoque de análisis muy común en estos casos (Davies, 2010; Starkman, 2014). En definitiva, son escasos los estudios que contrastan, desde el punto de vista de la cobertura periodística, las características informativas de los contenidos que se difunden antes y después de la explosión de las burbujas.

Estos rasgos generales de los estudios sobre la relación entre los medios y las burbujas, sobre todo financieras, son bastante comunes en la reciente investigación referida las burbujas inmobiliarias que alentaron las crisis económicas y financieras de 2008, sobre todo en países como Estados Unidos, Irlanda y España.

\section{Los medios y las burbujas inmobi- liarias}

En la ya abundante investigación sobre los medios y la crisis de 2008 (Schiffrin, 2011; Schifferes y Roberts, 2014; Lee, 2014; Picard, 2015) hay a menudo referencias parciales y someras a la cobertura periodística de la burbuja inmobiliaria. Predominan las menciones al hecho de que los medios alentaron la especulación en activos inmobiliarios. Por una parte, los medios se hallaban inmersos en una red de intereses societarios, publicitarios, etc., que les llevaba a favorecer a empresas constructoras y a aplaudir el optimismo de los mercados. Por otra parte, los periodistas fueron incapaces de comprender e interpretar la magnitud de los riesgos que acarreaba la extraordinaria escalada alcista de los precios de la vivienda, y su relación con la evolución de la economía real y financiera. La confluencia de estos dos factores es la base de muchos de los análisis que han tratado, aunque sea parcialmente, este tema.

En Estados Unidos, Starkman (2014) destaca cómo en términos generales los medios discutieron sobre la existencia de la burbuja, pero no llegaron a advertir el carácter corrupto de la estructura financiera montada en torno a las hipotecas subprime, ni las consecuencias sistémicas que podría tener el desplome inmobiliario. En España, Almiron (2008) concluye que la confluencia de 
los intereses económicos de los grupos mediáticos con los del sector de la construcción e inmobiliario impidió que los medios adoptaran una posición de alerta con respecto a la burbuja. Fahy, O'Brien y Poti (2010) plantean algo similar en el caso irlandés:

la tendencia de los periodistas financieros a operar en redes de comunicación elite-to-elite fue más pronunciada durante los años del Tigre Celta, cuando la falta de crítica por parte de reguladores, y fuentes económicas y políticas contribuyó a una carencia de discursos críticos en la cobertura económica de los medios (p. 18).

Son realmente muy minoritarios los trabajos que apuntan al hecho de que los medios $-\mathrm{O}$ al menos, algunos medios importantes- pudieron cumplir con su función de 'perro guardián' en los años previos al crash inmobiliario. Roush (2011), por ejemplo, defiende que fue así en el caso de los grandes periódicos estadounidenses, aunque su papel fue más bien el de Casandras, en un mercado que no deseaba escuchar malas noticias. Arrese (2015), refiriéndose a España, también apunta la idea de que los medios al menos mantuvieron viva en la opinión pública la discusión sobre la burbuja, y lo hicieron con un enfoque más equilibrado que el mantenido por muchos políticos y empresarios de la época.

Al margen de estas valoraciones generales, los pocos estudios específicos que existen sobre la cobertura periodística de la burbuja inmobiliaria arrojan luz sobre distintos aspectos del papel de los medios, así como de las fuentes y los protagonistas de las noticias, pero en términos generales no se distancian mucho de las ideas ya expuestas, sobre un cierto consenso general en torno a una cobertura fallida o deficiente.

Mercille (2014) ha estudiado con detalle la cobertura periodística de la burbuja inmobiliaria en Irlanda y ha concluido que antes del crash los medios publicaron muy pocas historias sobre la burbuja, y fueron en general muy reticentes a hacerse eco de los "profetas del desastre", adoptando posiciones vagas y ambivalentes sobre la existencia de la burbuja. También en torno al caso irlandés, Silke (2015) señala la explosión del sector inmobiliario como ejemplo de la íntima relación de dependencia entre los medios de comunicación y la economía de mercado:

La experiencia irlandesa confirma muchas de las teorías críticas sobre el rol de los medios en una sociedad capitalista por lo que se refiere a la relación entre economía, poder y política, y verifica también el papel largamente sospechado de los medios como una parte estructuralmente importante del moderno estado capitalista en lugar de ser el 'perro guardián' objetivo que vigila al poder (p. 315).

En este sentido, un análisis de contenidos llevado a cabo por Preston y Silke (2014) describe cómo los dos principales diarios nacionales irlandeses actuaron en buena medida como defensores del sector inmobiliario, un mercado en el que ambos periódicos tenían claros intereses. $\mathrm{Al}$ mismo tiempo, los autores 
detectaron "un clara tendencia a infravalorar cualquier anuncio de amenaza de un posible crash inmobiliario, defendiendo la idea de un 'aterrizaje suave' de los precios en su previsible corrección" (p. 22).

Desde una óptica distinta, focalizando el análisis en las fuentes utilizadas y en las posturas defendidas por economistas y expertos en los medios, Starr (2012) llega a la conclusión de que en torno a la burbuja inmobiliaria que se vivió en California el público fue suficientemente alertado de los peligros por parte de algunos economistas que intervinieron en los medios, aunque las posturas de estos expertos se vieron debilitadas por visiones contrarias de otros economistas con intereses en el sector:

Las previsiones negativas razonablemente formuladas por muchos economistas citados en los medios seguramente no tuvieron los efectos beneficiosos que podrían tener -al menos alertando sobre la burbuja que se estaba creando, o animando a muchos propietarios a cubrirse contra los riesgos que un desplome los precios pudiera acarrear-, porque se mezclaron con las opiniones muy optimistas sobre la evolución del sector realizadas por economistas en las nóminas de la industria inmobiliaria (p. 166).

En España, Arrese y Vara (2012) destacan el hecho de que en los años previos a la explosión de la burbuja la discusión sobre su existencia estuvo mediatizada por las posiciones políticas a favor o en contra de los distintos gobiernos -conservador y socialistaque tuvieron la responsabilidad de interpretar la extraordinaria escala de los precios de la vivienda que se estaba produciendo entre 2003 y 2007. Sin embargo, otros autores, como Müller (2011) y Illueca (2014) denuncian la superficialidad de la cobertura durante esos años, y la incapacidad de conectar el fenómeno inmobiliario con otros factores económicos y financieros que podían acarrear graves consecuencias para la economía del país. De hecho, Müller (2011) concluye que los medios españoles tuvieron un rol importante en la prolongación de la especulación que se vivió en el sector inmobiliario.

Otros trabajos abordan aspectos parciales de la relación entre la cobertura periodística y la evolución de los precios de la vivienda en esos años. Soo (2015), por ejemplo, analiza cómo la cobertura periodística de los medios locales sí permitía pronosticar adecuadamente las variaciones de precios y el volumen de actividad del sector durante los años del boom inmobiliario estadounidense, mientras que McCollough y Karani (2014) demuestran empíricamente cómo las noticias negativas sobre el sector pueden afectar a la percepción de los ciudadanos sobre el valor de las propiedades. Otros hallazgos coincidentes con esas mismas ideas son los que encontraron Glynn et al. (2008) en un análisis de contenidos de la cobertura por parte de los diarios estadounidenses de la evolución del sector inmobiliario entre 1996 y 2007, y de su correlación con los datos de 
encuestas entre los ciudadanos. Sus resultados indicaban que existe una clara relación entre la cantidad y el tipo de cobertura mediática sobre este tema y las percepciones de la ciudadanía sobre el estado del mercado de las viviendas. En contraste con esos trabajos, Walker (2014) muestra cómo en el caso británico la información sobre el mercado inmobiliario fue menos optimista a medida que los precios de la vivienda se incrementaban, algo que pudo explicar en parte que el crash inmobiliario en Gran Bretaña fuera menos servero en aquel país que en otros, como Estados Unidos. Por último, Landriscina (2012) estudió cómo la sección de noticias del sector inmobiliario del New York Times normalizó y legitimó los marcos de comprensión (frames) y los valores que se conectan con fenómenos problemáticos en torno al sector de la vivienda, como la generación de burbujas.

Ninguno de los estudios mencionados analiza en qué medida la forma de abordar periodísticamente el tema de la burbuja -desde el punto de vista de temas, fuentes, posiciones editoriales, etc.- se mantuvo o cambió sustancialmente, y en qué aspectos, en las fases de boom y crash inmobiliario. Mercille (2014) se refiere a la clara discrepancia entre la cobertura inmobiliaria antes y después de la explosión de la burbuja, pero sólo desde el punto de vista de la relevancia cuantitativa del tema. Ninguno, tampoco -salvo en cierta medida la investigación de Starr (2012)-, valora los cambios en los aná- lisis del fenómeno de la burbuja, en esos dos momentos, llevados a cabo por los principales agentes del sector (políticos, analistas, empresarios, etc.). El objetivo de este trabajo es precisamente complementar los estudios realizados hasta ahora con un enfoque longitudinal que tenga en cuenta esos temas de análisis, con la burbuja inmobiliaria española como base del estudio.

\section{La burbuja inmobiliaria en España}

Existen pocas dudas sobre el hecho de que la economía española sufrió una burbuja inmobiliaria que coincidió con las secuelas de la crisis financiera internacional. El Banco de España, tradicionalmente bastante reacio a utilizar la expresión "burbuja inmobiliaria", comparte esta idea en su análisis psoterior de la crisis. Por ejemplo, en su Boletín Económico de febrero de 2014 se puede leer que:

las probabilidades de un ajuste suave de la sobrevaloración de los precios y de la sobreproducción de vivienda se evaporó repentinamente en cuanto el mercado sufrió una corrección de una intensidad desconocida, lo que llevó a la economía española por primera vez en su historia reciente a la experiencia de un pinchazo de la burbuja inmobiliaria (Malo de Molina, 2014, p.16).

Los datos hablan por sí mismos. Desde su pico de máximo valor en 2008, los precios nominales de la vivienda cayeron un $30 \%$ en menos de cinco años (Gráfico 1). 
Gráfico 1. Precios nominales de la vivienda $(€ / \mathrm{m} 2)$

Junio $1995=1$

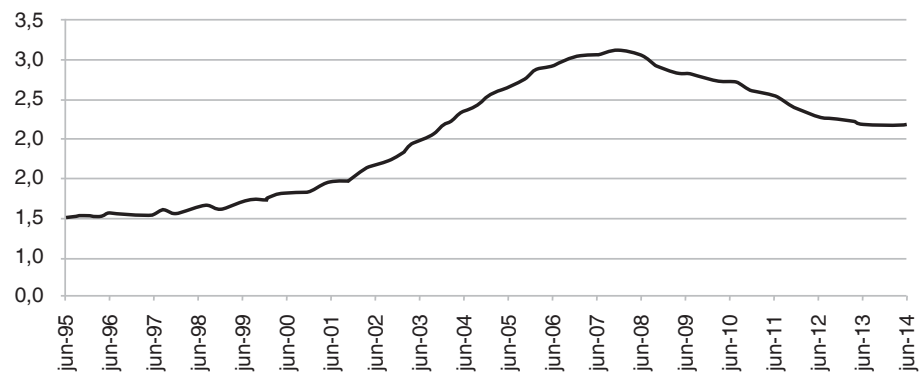

Fuente: Ministerio de la Vivienda de España

La burbuja de precios ocurrió al ñol en 2005 y 2006. Asimismo, supomismo tiempo que el sector de la cons- nía un $13 \%$ del mercado de trabajo, trucción experimentaba un extraordi- justo cuando éste estaba en sus niveles nario crecimiento, un verdadero más altos, antes de que comenzara la boom, al que siguió un profundo de- crisis. A finales de 2014, ambos datos, clive (Gráfico 2). De acuerdo con las participación en el PIB y en el mercaestadísticas oficiales, la construcción do laboral, habían caído hasta el 4,9\% representó casi un $11 \%$ del PIB espa- y 5,9\%, respectivamente.

Gráfico 2. Número de viviendas iniciadas Medias móviles trimestrales

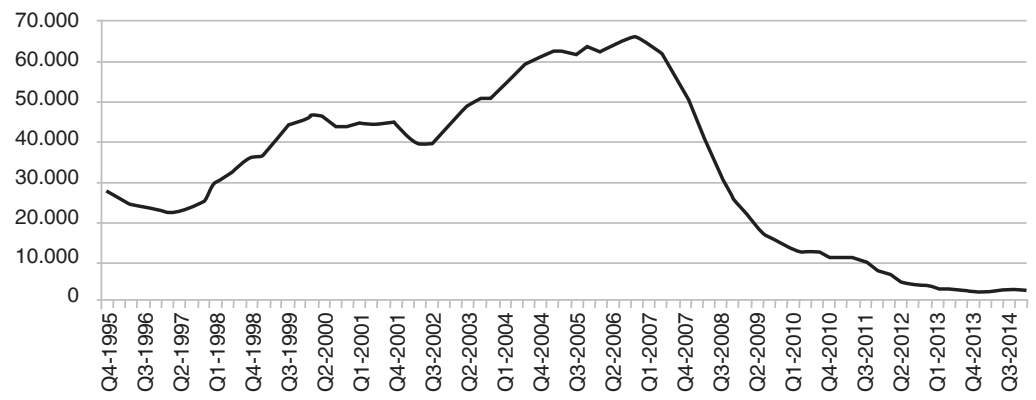

Fuente: Ministerio de la Vivienda de España 
La noción de burbuja no sólo depende de las expectativas de la gente sobre los precios futuros sino también de la ansiedad y de la preocupación por la posibilidad de enfrentarse a precios inaccesibles (Case y Shiller, 2003). No hay demasiados datos sobre cómo esas expectativas se comportaron y sobre cómo azuzaron la creación de la burbuja en el caso español. Sin embargo, García Montalvo (2006) ofrece los resultados de una encuesta llevada a cabo en cinco ciudades españolas en 2005: Madrid, Barcelona, Valencia, Murcia y La Coruña. De acuerdo con los resultados de esa encuesta, un 94,5\% de los encuestados estaban de acuerdo en que los precios estaban inflados $(40 \%$ de ellos consideraban que la sobrevaloración superaba el 50\%). Pero al mismo tiempo, la mayoría esperaba que los precios continuaran subiendo en los próximos 10 años, a un ritmo medio anual del $23 \%$.
Otra evidencia de la percepción sobre los precios la ofrece el Barómetro del CIS (Centro de Investigaciones Sociológicas), encuesta a la población española que mide mensualmente las opiniones y actitudes de los ciudadanos sobre temas de actualidad. El Barómetro incluye una pregunta sobre los tres problemas fundamentales que preocupan a los españoles. Como se observa en el Gráfico 3, la percepción de la vivienda como uno de esos problemas creció en importancia en el período 2000-2007. De hecho, en septiembre de 2007 era el problema que se percibía como más acuciante. A partir de ese momento, su valoración como problema fue decreciendo. La causa era evidente: el problema del desempleo pasó a ocupar la primera posición, como consecuencia del impacto adverso en la economía de la explosión de la burbuja.

Gráfico 3. Percepción pública de la vivienda como problema

$\%$ del total de respuesta

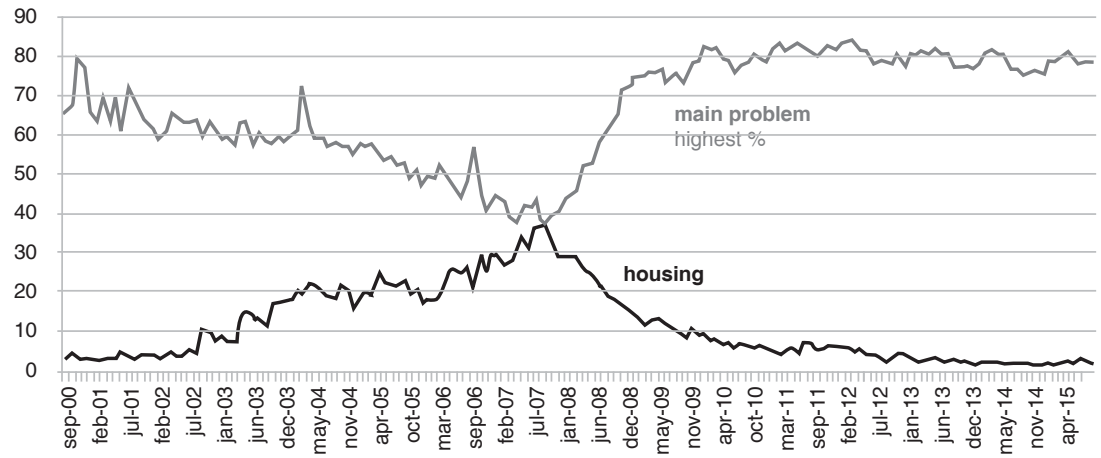

Fuente: Centro de Investigaciones Sociológicas 
Durante el largo período en el que los precios de la vivienda crecían y crecían, la detección de la existencia de una burbuja no fue fácil de realizar. Un incremento intenso de los precios bien podría haber sido una señal de alarma. Sin embargo, no suele ayudar mucho que se utilice la etiqueta de burbuja cada vez que los precios parecen crecer demasiado alto y demasiado rápido. $\mathrm{Al}$ mismo tiempo, tampoco suele ser muy realista asumir que la evolución de los precios sea siempre el resultado de un mercado teóricamente competitivo y eficiente, sin lugar para la creación de esas burbujas.

Por lo que se refiere al discurso de los medios sobre la burbuja, García Montalvo (2008) ha comentado:

Siempre que existe un fenómeno complejo e impreciso, o una llaga donde no se pueda poner el dedo, se forman bandos. En el tema de la burbuja inmobiliaria también han existido al menos dos bandos: los negacionistas, que renegaban de que pudiera existir una burbuja, y los burbujistas, que sostenían la opinión contraria. La correlación de fuerzas entre estos grupos fue cambiando con el tiempo y sus contiendas más importantes tuvieron lugar principalmente en los medios de comunicación y en los foros de Internet (p. 10).

Case y Shiller (2003) también destacan a los medios como un foro privilegiado de discusión de la existencia o no de la burbuja inmobiliaria:

La prensa popular está llena de especulaciones sobre la posibilidad de que en Estados Unidos, y en otros países, exista una burbuja inmobiliaria que va a explotar. Barrons, la revista Money y The Economist han llevado a portada recientemente historias sobre la escalada irracional de los precios de la vivienda y sobre un posible crash. The Economist ha publicado una serie de artículos con títulos como 'Castles in Hot Air,' 'House of Cards,' 'Bubble Trouble,' and 'Betting the House.' Estas informaciones necesariamente han generado preocupación en el público en general (p. 299).

Tras el colapso de la burbuja, la economía española entró en una fase de fuerte recesión, el nivel desempleo se elevó por encima del $20 \%$ y algunas entidades financieras tuvieron que ser rescatadas finalmente con fondos públicos. Por supuesto, el sector de la construcción e inmobiliario sufrieron especialmente las fatales consecuencias de la crisis, aunque quizá no con el impacto catastrófico que habían augurado muchos expertos. De hecho, en 2010, un especialista en crisis financieras como Edward Chancellor escribía en el Financial Times:

España es quizá el ejemplo de burbuja inmobiliaria que más perplejidad causa. Durante el largo boom económico, la península ibérica se inundó de proyectos de construcción especulativos. El sector llegó a suponer un $18 \%$ del PIB. El ratio entre los precios de la vivienda y la renta disponible ascendió de 3,8 veces a finales de los años noventa a 7,7 veces en 2007. Y a pesar de la severidad de la crisis, que llevó al desempleo por encima del 20\%, el mercado de viviendas residenciales se 
ha demostrado especialmente resiliente. (...) Tras doblarse en la primera mitad de la década, los precios de la vivienda han caído sólo un $15 \%$ en términos reales. El ratio entre el precio de la vivienda y la renta permanece aún un $80 \%$ por encima de su valor medio a largo plazo. No está claro por qué los precios de la vivienda en España se han mostrado tan inflexibles (Chancellor, 2010).

\section{Estudio empírico: análisis de conte- nido}

El presente estudio analiza las posiciones sobre la burbuja inmobiliaria mantenidas por periodistas, políticos y economistas en cinco diarios españoles (los tres diarios líderes de ámbito nacional -El País, El Mundo y ABC-, y los dos principales diarios económicos Expansión y Cinco Días-). De los cinco periódicos, dos de ellos tienen una orientación política más orientada al centro izquierda (El País y Cinco Días), y el resto se pueden enmarcar en el ámbito ideológico del centro derecha $(E l$ Mundo, $A B C$ y Expansión). Esta selección de diarios se ha demostrado eficiente en diferentes estudios que han tratado de analizar la opinión pública en España sobre cuestiones económicas y otros temas especializados a nivel nacional (Elías, 2001; De Miguel \& Pozas, 2009; Arrese, 2015b).

Por lo que se refiere al enfoque metodológico, en esta investigación se utiliza el análisis de contenido cuantitativo, enfocado en las divergencias en las opiniones de políticos, expertos y la propia voz de los medios en torno a la naturaleza, la estructura y los marcos de interpretación de la burbuja inmobiliaria en dos períodos temporales claramente diferenciados: de 2003 a 2007, cuando se produjo un vivo y continuado debate en la prensa sobre si se estaba formando o no una burbuja en el sector inmobiliario; y de 2008 a 2013, cuando todo el mundo reconocía abiertamente que la explosión de la burbuja se había producido, y que era la causante principal de la crisis económica y financiera que se viviría en el país.

La hipótesis general de la investigación es que antes de la crisis no hubo una discusión real sobre la amenaza de una burbuja inmobiliaria, sobre sus causas y posibles consecuencias, sino más bien un orquestado consenso 'negacionista' -especialmente entre la clase política-. Sólo después del pinchazo de la burbuja, los mismos políticos y los expertos hicieron un esfuerzo real por explicar las causas y las consecuencias del extraordinario incremento de los precios de la vivienda en la última década, pero de nuevo, el análisis fue distorsionado por el frame de la responsabilidad (por la discusión sobre quién había sido el responsable o los responsables de la situación). El estudio explora las diferencias entre las opiniones de los políticos, expertos y periodistas en los dos períodos analizados, así como las diferencias entre los diarios según su enfoque editorial (generalista o especializado, y más hacia la derecha o más hacia la izquierda del espectro ideológico).

La investigación se basa en los artículos extraídos de la base de datos Factiva, que ofrece el acceso al texto completo 
de los contenidos de las versiones impresas de los cinco diarios en el período de tiempo analizado. La primera extracción de artículos incluyó todos los trabajos con la expresión "burbuja AND inmobiliaria” en cualquier parte del texto: titular, sumario o texto principal del artículo. Esa primera muestra de 6.872 artículos fue utilizada para analizar la intensidad de la cobertura sobre la burbuja inmobiliaria entre 2003 y 2013. Dentro de esa base de artículos, posteriormente se seleccionaron aquellos que mencionaban la "burbuja inmobiliaria" en el titular (275 artículos), lográndose una muestra de trabajos que ofrecían una discusión sustancial del tema y respuestas a gran parte de las preguntas planteadas en el libro de códigos y el cuestionario del análisis de contenidos, que se explica a continuación. Esta fue la base de textos utilizada para el estudio.

Las variables utilizadas para la investigación y descritas en el libro de codificación fueron seleccionadas utilizando un proceso de acercamiento deductivo e inductivo al tema de estudio (Neuendorf, 2002). En primer lugar se identificaron deductivamente las variables que se debían tener en cuenta a través de la revisión de las principales cuestiones analizadas al considerar la cobertura mediática de las crisis económicas y financieras, y en especial las inmobiliarias, en los trabajos analizados en la revisión de la literatura. A partir de ellas, se elaboró una hoja de códigos con las preguntas básicas en torno a la burbuja (existencia o no de la burbuja, causas, medidas propuestas ante la es- calada de precios, consecuencias positivas y negativas, responsables, etc.), y con las cuestiones fundamentales sobre su cobertura periodística (tipo de artículos, autores, características de las fuentes, tipo de periódico, etc.). Esta primera hoja de codificación fue aplicada a una muestra aleatoria de los artículos seleccionados. En esta fase inductiva, se examinó qué variables podrían ser añadidas al análisis, y cuáles de las existentes podrían ser modificadas o eliminadas. La plantilla de códigos fue revisada en tres ocasiones hasta que finalmente se aprobó la versión que se aplicaría en el test de fiabilidad y concordancia entre codificadores.

Los artículos fueron analizados por los dos autores del estudio y por dos codificadores más entrenados en el análisis de contenido, aplicando la hoja de códigos final compuesta por 30 variables. La codificación tuvo lugar entre Enero y Marzo de 2017. Se llevó a cabo en primer lugar un test de fiabilidad entre codificadores (ICR) aplicado a un $10 \%$ de los artículos seleccionados aleatoriamente, cuyo resultado mostró valores aceptables, con alphas de Krippendorff que se situaron entre .76 y 1 para las diferentes variables. Tras finalizar ese proceso, los autores del estudio revisaron el libro de códigos y las discrepancias principales en algunas de las variables, y tras su análisis y discusión, aprobaron el cuestionario final. Éste fue aplicado de nuevo a una muestra aleatoria de 20 artículos, lográndose una mejora de los alphas de Krippendorff, que pasaron a situarse en el rango .81-1. 


\section{Resultados y hallazgos}

El primer hallazgo que cabe destacar a partir de los resultados de la investigación es que el concepto de burbuja inmobiliaria fue ampliamente usado en las noticias, tanto antes como después de su estallido. Casi 7.000 artículos mencionaron la burbuja entre 2003 y 2007 en los cinco periódicos analizados, lo que supone una media de algo más de 100 artículos por periódico y año. Al ser un concepto bastante técnico, este nivel de uso -en torno a un artículo cada cuatro días en cada periódico, por término medio- se puede considerar elevado. Así pues, es claro que las referencias a la existencia o no de una burbuja inmobiliaria fueron muy comunes en la opinión pública a lo largo de todo el período. Por supuesto, los datos también indican que, en términos de intensidad, la cobertura se incrementó sustancialmente en el período 2008-2013, tras el pinchazo de la burbuja. Aunque el número de artículos ascendió a 1.614 antes de 2008, la cantidad casi se triplicó hasta los 5.258 en el período 2008-2013 (véase Gráfico 4). Asimismo, es interesante hacer notar que antes de la explosión de 2008, hubo un año -el año 2003- en el que la referencia a la posibilidad de que hubiera una burbuja inmobiliaria en España fue especialmente intensa en la prensa, una cobertura del tema que posteriormente se moderó mucho.

Este primer análisis genérico es importante porque indica que la cobertura periodística de la burbuja inmobiliaria y, como consecuencia, la discusión y debate sobre su existencia fue bastante relevante durante los años analizados. $\mathrm{Al}$ mismo tiempo, es interesante observar cómo el patrón de esa cobertura en el caso español, que se ajusta prácticamente a una curva en forma de S, con un pico inicial en 2003 y con un claro punto de inflexión en 2008, es muy similar, por ejemplo, a la evolución descrita por Mercille (2014) para el caso de la cobertura de la burbuja inmobiliaria irlandesa.

Gráfico 4. Artículos que mencionan la burbuja inmobiliaria (2003-2013)

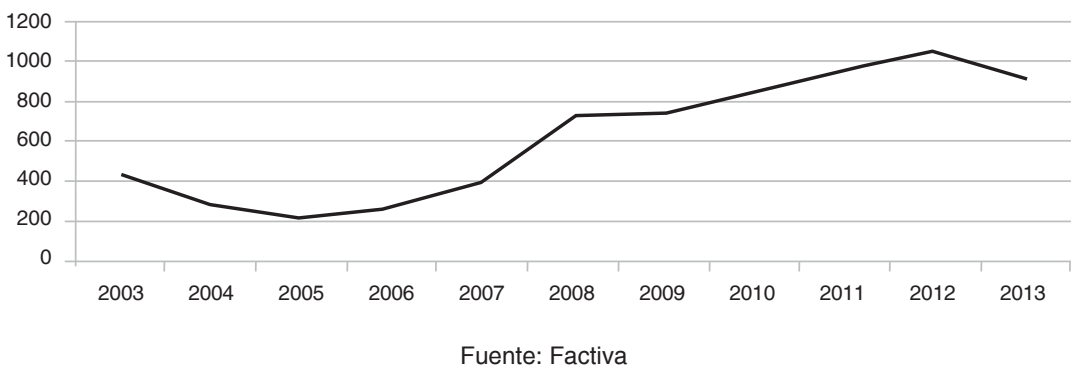


La evolución de la cobertura es muy similar a la del Gráfico 4 si se tienen en cuenta los artículos considerados para el análisis de contenidos -aquellos que incluyen la expresión burbuja inmobiliaria en el titular-, aunque en este caso hay relativamente una mayor presencia del tema en las noticias en los primeros años, 2003 y 2004. Tomando ya como referencia esa muestra de artículos del análisis de contenidos, a continuación se comentan los principales resultados del estudio.

Por lo que se refiere a la primera cuestión estudiada -las opiniones sobre la existencia o no de la burbuja-, la discusión sobre este asunto fue muy intensa en el primer período del análisis, entre 2003 y 2007 , cuando muchos medios y organizaciones internacionales alertaban sobre el problema de la burbuja inmobiliaria española, tal y como informaban los propios medios españoles. En términos generales, se puede afirmar que los diarios estudiados publicaron más opiniones y juicios que reflejaban visiones pro-burbuja que sus contrarias.
Durante el período, 128 artículos contenían juicios explícitos o implícitos sobre el tema, de los que $86(65 \%)$ apoyaban la tesis de que existía una burbuja y 45 (35\%) la de que no se podía hablar de tal fenómeno. Por supuesto, como cabía esperar, ese mismo análisis en el período 2008-2013 daba un resultado muy diferente: de 174 referencias a esa cuestión, 172 (99\%) daban por hecho que se había producido una burbuja inmobiliaria, y sólo 2 (15) dudaban de que lo sucedido pudiera catalogarse de esa forma.

$\mathrm{Al}$ considerar la naturaleza de las opiniones expresadas cuando existía debate sobre la existencia de la burbuja, la Tabla 1 muestra cómo se produjo una clara polarización entre fuentes 'negacionistas' -utilizando la terminología de García Montalvo (2008)-, compuestas fundamentalmente de políticos, funcionarios de la administración pública, directivos de banca y otras entidades financieras, y representantes del sector inmobiliario y de la construcción, y fuentes 'burbujistas' (sobre todo expertos y medios de comunicación internacionales).

Tabla 1. ¿Existe una burbuja inmobiliaria en España? (2003-2007)

\begin{tabular}{|l|l|l|c|}
\hline \multicolumn{1}{|c|}{ Fuentes } & \multicolumn{1}{c|}{ Sí } & \multicolumn{1}{c|}{ No } & Total \\
\hline Negacionistas & $13(29,5 \%)$ & $31(71,5 \%)$ & $44(100 \%)$ \\
\hline Burbujistas & $29(72,5 \%)$ & $11(27,5 \%)$ & $40(100 \%)$ \\
\hline Total & $\mathbf{4 2 ( 5 0 \% )}$ & $\mathbf{4 2 ( 5 0 \% )}$ & $\mathbf{8 4 ( 1 0 0 \% )}$ \\
\hline
\end{tabular}

Los datos anteriores se completan con las opiniones o juicios mantenidos en los diarios por sus propios profesionales (artículos firmados por periodistas, por el propio medio, editoriales, etc.), cuando en ellos se mostraban claras opiniones no asignables a fuentes externas específicas. En este caso, el resultado es claramente más extremo: la gran mayoría de las posturas explícitas o implícitas de los artículos sobre la existencia de la burbuja -41 de 44- eran positivas. 
La prevalencia de esa visión 'burbujista' en los periódicos analizados, entre 2003 y 2007, no estaba influenciada significativamente por el tipo de periódico considerado. Tanto los diarios generalistas (El País, El Mundo y ABC) como los económicos (Expansión y Cinco Días) publicaron más opiniones y análisis alineados con la hipótesis de la existencia de la burbuja que con su contraria. Aunque la visión 'negacionista' fue relativamente mayor en la prensa económica (31 de 73 artículos) que en la generalista (14 artículos de 55), esa diferencia se encuentra en el límite de la significación estadística $\left(X^{2}(1, \mathrm{~N}=128)=3.981, p=0.045\right)$. Al mismo tiempo, es interesante advertir que de los 275 artículos analizados, en los que la expresión "burbuja inmobiliaria" aparece en el titular, prácticamente la mitad (140) se publicaron en los dos diarios con una orientación ideológica más hacia la izquierda, El País y Cinco Días.

El predominio cuantitativo de las posturas 'burbujistas' debe interpretarse, sin embargo, en el contexto de la relevancia cualitativa de las visiones 'negacionistas'. Entre 2003 y 2007, tanto los Ministros de Economía Socialistas como Populares, los gobernadores del Banco de España, y los principales empresarios y banqueros del país negaron una y otra vez la existencia de la burbuja. Además, sus rechazos fueron explícitos y directos ("En España no hay una burbuja inmobiliaria”), mientras que las posturas de los que defendían una idea contraria a menudo eran más ambiguas ("No deberíamos desestimar los riesgos de existencia de una burbuja inmobiliaria").

Por otra parte, el marco de discusión sobre la burbuja inmobiliaria en ese período previo a su explosión fue muy simple, centrado casi exclusivamente en las causas del extraordinario incremento de los precios de la vivienda, pero con muy pocas referencias a las posibles consecuencias de su pinchazo, y menos todavía al análisis de responsabilidades. Como se muestra en la Tabla 2, tras el desplome de los precios, los marcos de explicación que se utilizaban en artículos fueron más complejos y equilibrados, con una creciente atención a las consecuencias de la explosión de la burbuja y a las responsabilidades en torno a la crisis. Para valorar los datos de la Tabla 2, hay que tener en cuenta que en cada artículo analizado se registraron hasta tres causas, tres consecuencias y tres responsables citados en el texto.

Tabla 2. Referencias a causas, consecuencias y responsabilidades en torno la burbuja inmobiliaria antes y después de su explosión

\begin{tabular}{|l|r|r|r|}
\hline & \multicolumn{1}{|c|}{$\mathbf{2 0 0 3 - 2 0 0 7}$} & \multicolumn{1}{|c|}{$\mathbf{2 0 0 8 - 2 0 1 3}$} & \multicolumn{1}{c|}{ Total } \\
\hline Causas & $143(67,4 \%)$ & $108(31,4 \%)$ & $251(45,1 \%)$ \\
\hline Consecuencias & $43(20,3 \%)$ & $163(47,4 \%)$ & $206(37,1 \%)$ \\
\hline Responsabilidades & $26(12,3 \%)$ & $73(21,2 \%)$ & $99(17,8 \%)$ \\
\hline Total & $\mathbf{2 1 2 ( 1 0 0 \% )}$ & $\mathbf{3 4 4 ( 1 0 0 \% )}$ & $\mathbf{5 5 6 ( 1 0 0 \% )}$ \\
\hline
\end{tabular}


Por lo que se refiere a las fuentes citadas en los artículos, la mezcla de ellas es bastante similar en los dos períodos analizados, aunque hay que destacar también algunas peculiaridades. La
Tabla 3 describe los tipos de fuentes utilizadas antes y después del pinchazo de la burbuja, teniendo en cuenta que en cada artículo se podían registrar hasta tres fuentes citadas.

Tabla 3. Fuentes citadas en los artículos sobre la burbuja inmobiliaria española

\begin{tabular}{|l|r|r|r|}
\hline & \multicolumn{1}{|c|}{$\mathbf{2 0 0 3 - 2 0 0 7}$} & \multicolumn{1}{c|}{$\mathbf{2 0 0 8 - 2 0 1 3}$} & \multicolumn{1}{c|}{ Total } \\
\hline Gob., adm. y pol. & $24(21,1 \%)$ & $34(19,6 \%)$ & $58(20,2 \%)$ \\
\hline Finanzas y negocios & $55(48,2 \%)$ & $73(42,2 \%)$ & $128(44,6 \%)$ \\
\hline Econom. y expertos & $26(22,8 \%)$ & $33(19,1 \%)$ & $59(20,5 \%)$ \\
\hline Sociedad & $2(1,7 \%)$ & $28(16,2 \%)$ & $30(10,5 \%)$ \\
\hline Otros medios & $7(6,2 \%)$ & $5(2,9 \%)$ & $12(4,2 \%)$ \\
\hline Total & $\mathbf{1 1 4 ( 1 0 0 \% )}$ & $\mathbf{1 7 3 ( 1 0 0 \% )}$ & $\mathbf{2 8 7 ( 1 0 0 \% )}$ \\
\hline
\end{tabular}

Como se puede observar en la Tabla 3, una diferencia sobresale entre todas las demás al comparar los dos períodos de análisis: el gran incremento de fuentes de la sociedad (ciudadanos, organizaciones sin ánimo de lucro, asociaciones de consumidores, etc.) en el período 2008-2013. Por supuesto, tras la explosión de la burbuja la ciudadanía emergió como la gran afectada por la crisis, algo que no se tuvo en cuenta en absoluto en los años del boom inmobiliario. Por lo que se refiere al resto de los actores, el peso de los distintos tipos de fuentes (Gobierno, administración y política; Finanzas y negocios; Economistas y expertos; Otros medios periodísticos) se mantiene bastante estable a lo largo de todo el período, aunque como ya se ha explicado, sus opiniones y marcos de análisis fueron distintos antes y después de la explosión de la burbuja. De hecho, considerada de forma global, la mezcla de fuentes en esos dos períodos no se modifica de una forma estadística significativa $\left(X^{2}(4, \mathrm{~N}=277)=9.859, p=0.042\right)$, a pesar de esa gran disparidad en las voces procedes de la sociedad.

Finalmente, al valorar la diferencia de cobertura entre distintos tipos de periódicos, la prueba Chi cuadrado no ofrece resultados estadísticamente significativos al comparar la mezcla de fuentes utilizadas en los dos períodos ni entre la prensa generalista y la econó$\operatorname{mica}\left(X^{2}(4, \mathrm{~N}=277)=8.611, p=0.071\right)$ ni entre los periódicos agrupados por $\mathrm{su}$ orientación ideológica $\left(X^{2}(4\right.$, $\mathrm{N}=277)=0.378, p=0.984)$. Esa misma prueba sí detecta diferencias significativas comparando esos mismos grupos de diarios por su referencia a las causas, consecuencias y responsables de la burbuja. En este caso, la prensa generalista ofrece un marco de explicación más amplio y variado que la económica $\left(X^{2}(2, N=546)=24.312, p<0.01\right)$, y la de orientación más hacia la izquierda del 
espectro político pone un mayor énfasis en el análisis de las causas de la burbuja que la prensa de centro-derecha $\left(X^{2}(2, N=546)=18.487, p<0.01\right)$.

En síntesis, los resultados del análisis estadístico permiten concluir que la cobertura de la crisis inmobiliaria española por parte de la prensa española dio en los primeros años analizados suficiente voz a quienes mantenían que existía una burbuja, aunque al mismo tiempo se escucharon con fuerza las posturas de influyentes líderes políticos y económicos que negaban ese hecho. Antes de la explosión, los periodistas y expertos expresaron claras dudas sobre esa versión oficial, que mantenía que el extraordinario crecimiento de los precios estaba justificado por las condiciones económicas. Sin embargo, ni los medios ni los expertos, fueron capaces de encuadrar la situación en un escenario de alerta suficientemente claro en el que el riesgo y la urgencia activaran la necesaria acción pública. Sólo tras el pinchazo, los periódicos prestaron una mayor atención a todas las dimensiones del colapso inmobiliario, cubriendo de forma más profunda sus efectos y las responsabilidades políticas, económicas y financieras. Más allá de una atención más intensa por parte de los medios de orientación de centro-izquierda, el patrón general de la cobertura periodística de este tema fue muy similar en los distintos tipos de periódicos considerados en este trabajo.

\section{Discusión}

"Tuvimos ocho años para pinchar la burbuja y no lo hicimos" (El País, 2011). Así expresaba un mea culpa colectivo en julio de 2011 el candidato socialista a la presidencia del gobierno, Alfredo Pérez Rubalcaba, cuando fue preguntado si había algo de lo que se arrepentía de su experiencia como Ministro en los gobiernos de José Luis Rodríguez Zapatero, entre 2004 y 2011. Hoy, las consecuencias de la burbuja inmobiliaria española en la vida de millones de españoles continúan percibiéndose como profundas y dramáticas. De hecho, en febrero de 2017 el parlamento español decidió establecer una Comisión de Investigación para la analizar las causas, consecuencias y responsabilidades la crisis económica y financiera que se activó tras el pinchazo de la burbuja. En esa comisión, en enero de 2018, el Ministro de Economía Luis de Guindos -que también había sido Secretario de Estado de Economía en el período 2000-2004volvía a reconocer: "La burbuja inmobiliaria, la burbuja crediticia y la pérdida de competitividad fueron las causas que llevaron a España a la peor crisis financiera y económica de su historia moderna”. No es extraño que una década después de su estallido, el concepto siga muy vivo en la mente de la ciudadanía, y esa sensibilidad y el miedo a experimentar situaciones parecidas haga que en los medios se utilice la expresión ante cualquier signo de alza excesiva de precios de la vivienda (“¿Otra burbuja? Moody's prevé una subida anual del precio de la vivienda de casi el $5 \%$ hasta 2019”, Cinco Días, 24 May 2017).

Entender mejor el papel de los medios de comunicación en la formación y evolución de burbujas, como en el caso de la burbuja inmobiliaria española, es 
una tarea importante para los investigadores que intentan contribuir a la mejora del trabajo de los periodistas y las redacciones que cubren la actualidad en momentos de incertidumbre económica y financiera. Desde este punto de vista, de la presente investigación, y del análisis de contenidos llevado a cabo, se derivan algunas ideas que merecen ser destacadas:

1. Funciones de los medios. $\mathrm{Al}$ menos en el caso de España, no se puede afirmar que la prensa desatendiera la cobertura del problema de la burbuja durante los años previos a su estallido, aunque una postura más combativa, una atención más continuada y una mayor focalización en los signos de alarma podrían haber ayudado a hacer más conscientes a las autoridades y a la ciudadanía sobre las consecuencias previsibles de un colapso de los precios. Esta conclusión está más en línea con los hallazgos de Roush (2011) para el caso de Estados Unidos, que con los resultados de otras investigaciones sobre países que sufrieron un fenómeno similar, como el caso irlandés (Mercille, 2014).

2. Discurso oficial. En contextos de posibles generaciones de burbujas, son necesarios mayores esfuerzos por parte de los medios para desafiar públicamente el consenso oficial que a menudo emerge en torno a temas económi$\cos y$ financieros controvertidos, sobre todo cuando ese consenso contrasta con la percepción de sentido común de la realidad (la mayoría de los españoles en los años del boom sabían que la espiral de precios al alza no se podía mantener mucho tiempo) y con las llamadas de alerta de medios y organismos internacionales, tales como el Fondo Monetario Internacional y la OCDE. Un componente importante de ese esfuerzo debería ser la ampliación de la mezcla de fuentes que se utilizan en períodos de gran incertidumbre sobre temas complejos, como la evolución de los precios en situaciones de boom económico y financiero.

3. Expertos independientes. Como en el caso de la crisis de la vivienda en California (Starr, 2012), la mayoría de los economistas y expertos independientes utilizados como fuentes en los medios españoles mostraron dudas y críticas antes la tesis 'negacionista', y su presencia fue relativamente importante en ciertos momentos. Sin embargo, no hubo voces expertas consistentes, regulares y con autoridad que pudieran equilibrar y retar a las sólidas posiciones 'negacionistas' mantenidas por líderes e instituciones como el Ministerio de Economía, el Banco de España o bancos como el Banco de Santander. En tales circunstancias, los medios deben asegurarse que las posturas críticas con las voces oficiales ganen peso y autoridad en sus contenidos, sobre todo dando protagonismo a los expertos independientes más cualificados.

4. Contextos y escenarios. Como ya se ha comentado, el debate sobre la existencia de la burbuja no fue lo suficientemente profundo como para activar una discusión sobre los posibles efectos a corto, medio y largo plazo de su estallido. El marco de análisis se circunscribió a la explicación de las posibles causas del extraordinario crecimiento de 
los precios de la vivienda, pero no se abordó la consideración de las consecuencias posibles de escenarios futuros (aterrizaje suave, reajuste controlado, estallido repentino, etc.) y tampoco se acudió a la contextualización de la situación española tomando como referencia la historia, pasada o reciente, de otras burbujas inmobiliarias o de activos, como la que sufrió Japón a finales de 1991 y principios de 1992, sólo por un citar un ejemplo. Este esfuerzo de contextualización, tanto en el tiempo (corto y largo plazo, y en la historia) como en el espacio (otras experiencias internacionales), es muy necesario para que los periodistas económicos sean capaces de comprender mejor la lógica de la economía y de los mercados en períodos de boom y de crash, y en especial durante crisis financieras y de activos como los inmobiliarios.

\section{Bibliografía}

Almiron, N. (2008). Crisis financiera, economía y medios de comunicación. Mientras Tanto, 108-109, pp. 83-90.

Akerlof. G. A. y Shiller. R. (2009). Animal Spirits: How Human Psychology Drives the Economy, and Why It Matters for Global Capitalism. Princeton, NJ: Princeton University Press.

Arlt, H.-J. y Storz, W. (2010). Wirtschaftsjournalismus in der Krise. Frankfurt/Main: Otto Brenner Stiftung.

Arrese, Á. (2010). Periodismo económico, entre el boom y el crash (pp. 17-27). En: Arrese, A., Etayo, C., Moreno, E., Guerrero, E. y Navarro, M. (eds.). Periodismo económico: viejos y nuevos desafíos. Pamplona: Eunsa.

Arrese, Á. y Vara, A. (2012). ¿Canarios en la mina? La prensa y los riesgos de la "burbuja inmobiliaria" en España. Comunicación y riesgo. III Congreso Internacional de la Asociación Española de Investigación en Comunicación, AE-IC, Tarragona, December 20. http://dadun.unav.edu/bitstream/10171/22247/1/comunicacion\%20definitiva.pdf

Arrese, A. y Vara, A. (2014). Alarma y alarmismo: medios de comunicación y crisis económica. Estudios sobre el Mensaje Periodístico, 20 (2), pp. 933-951.

Arrese, A. (2015a). Spanish Press. No Illusions (p. 87-102). En: Schifferes, S. y Roberts, R. (eds.) The Media and Financial Crisis. London: Routledge.

Arrese, A. (2015b). Euro crisis metaphors in the Spanish press. Communication and Society, 28 (2), pp. 19-38.

Augey, D. y Brin, C. (2004). Media and Manias: News and Economic Decision-Making. Paper presented at the 6th World Media Economics Conference, HEC Montreal, May 12-15. http://www.cem.ulaval.ca/pdf/augey_brin.pdf

Barber, B. M. y Odean, T. (2008). All that glitters: The effect of attention and news on the buying behavior of individual and institutional investors. The Review of Financial Studies 21 (2), pp. 785-818. doi: https://doi.org/10.1093/rfs/hhm079

Bhattacharya, U., Galpin, N., Ray, R. y Yu, X. (2009). The Role of the Media in the Internet IPO Bubble. Journal of Financial and Quantitative Analysis 44 (3), pp. 657-682. doi: https:// doi.org/10.1017/S0022109009990056 
Brunnermeier, M. K. (2008). Bubbles. En Durlauf, S. N. y Blume, L. E. (eds.). The New Palgrave Dictionary of Economics. Second Edition. London: Palgrave Macmillan.

Campbell, G., Turner, J. D. y Walker, C. B. (2012). The role of media in a bubble. Explorations in Economic History, 49, pp. 461-481. doi: https://doi.org/10.1016/j.eeh.2012.07.002

Case, K. y Shiller, R. (2003). Is there a bubble in the housing market? Brooking Papers on Economic Activity 2, pp. 299-362. doi: https://doi.org/10.1353/eca.2004.0004

Chan, W. S. (2003). Stock price reaction to news and to no-news: Drift and reversal after headlines. Journal of Financial Economics, 70, pp. 223-260.

Chancellor, E. (2000). Devil Take the Hindmost: A History of Financial Speculation. New York: Plume.

Chancellor, E. (2010). Why some housing bubbles remain. Financial Times, 3 October 2010.

Dale, R. (2004). The First Crash. Princeton, NJ: Princeton University Press.

Davies, H. (2010). The Financial Crisis. Who is to Blame? London: Polity Press.

De Miguel, J. C. y Pozas, V. (2009). ¿Polarización ideológica o económica? Relaciones entre los medios y el poder político y corporativo. Viento Sur, 103, pp. 43-51.

Dougal, C., Engelberg, J., García, D. y Parsons, C. A. (2012). Journalists and the Stock Market. Review of Financial Studies 25 (3), pp. 639-679. doi: https://doi.org/10.1093/rfs/ hhr 133

Dyck, A. y Zingales, L. (2003). The Bubble and the Media. En Cornelius, P. y Kogut, B. (eds.). Corporate Governance and Capital Flows in a Global Economy (pp. 83-104). New York: Oxford University Press.

El País (2011). "Rubalcaba: 'Tuvimos ocho años para pinchar la burbuja y no lo hicimos'. El País, 31 de julio de 2011.

Elías, C. (2001). Periodismo especializado en medioambiente: el caso Doñana como paradigma de manipulación informativa. Ámbitos, 6, pp. 279-303.

Ellis, A. (1892). Influence of opinion on markets. The Economic Journal 2 (5), pp. 109-116. doi: https://doi.org/10.2307/2955943

Engelberg, J. y Parsons, C. A. (2011). The causal impact of media in financial markets. Journal of Finance, 66 (1), pp. 67-97. doi: https://doi.org/10.1111/j.1540-6261.2010.01626.x

Fahy, D., O’Brien, M. y Poti, V. (2010). Combative Critics or Captured Collaborators? Irish Financial Journalism and the End of the Celtic Tiger. Irish Communications Review, 12, pp. 5-21.

Fang, L. y Peress, J. (2009). Media coverage and the cross-section of stock returns. The Journal of Finance, 64 (5), pp. 2023-2052. doi: https://doi.org/10.2139/ssrn.971202

Galbraith, J. K. (1955). The Great Crash 1929. Boston: Houghton Mifflin Harcourt.

Garber, P. M. (1989). Tulipmania. Journal of Political Economy, 97 (31), pp. 535-560.

Garber, P. M. (2000). Famous First bubbles: The Fundamentals of Early Manias. Cambridge, MA: MIT Press.

García Montalvo, J. (2006). Deconstruyendo la burbuja: expectativas de revalorización y precio de la vivienda en España. Papeles de economía española, 109, pp. 44-75.

García Montalvo, J. (2008). De la quimera inmobiliaria al colapso financiero. Crónica de un desenlace anunciado. Barcelona: Antonio Bosch. 
García Montalvo, J. (2009). Los orígenes inmobiliarios de la crisis económica actual. En Cabrales, A. et al. La Crisis de la Economía Española (pp. 32-36). Madrid: Sociedad Abierta-Fedea.

Glynn, C. J., Huge, M. E. y Hoffman, Li. (2008). Did the media help inflate the housing bubble? Media coverage of real estate markets in times of change. Paper, Annual Conference of the AEJMC, April, 2008.

Illueca, M. (2014). Medios de comunicación y crisis financieras: ¿jpor qué nadie nos avisó? Cuadernos de Periodistas, 27, pp. 81-93.

Kalogeropoulos, A., Svensson, H. M., van Dalen, A., de Vreese, C. y Albaek, E. (2015). Are watchdogs doing their business? Media coverage of economic news. Journalism, 16 (8), pp. 993-1009. doi: https://doi.org/10.1177/1464884914554167

Kindleberger, C. P. (1978). Manias, Panics, and Crashes: A History of Financial Crises. New York: John Wiley and Sons.

Kleinnijenhuis, J., Schultz, F., Oegema, D. y van Atteveldt, W. (2013). Financial news and market panics in the age of high-frequency sentiment trading algorithms. Journalism, 14 (2), pp. 271-291. doi: https://doi.org/10.1177/1464884912468375

Knowles, S., Phillips, G. y Lidberg, J. (2017). Reporting the Global Financial Crisis. A longitudinal tri-nation study of mainstream financial journalism. Journalism Studies, 18 (3), pp. 322-340. doi: https://doi.org/10.1080/1461670X.2015.1058182

Kurtz, H. (2000). The Fortune Tellers. Inside Wall Street's Game of Money, Media, and Manipulation. New York: The Free Press.

Landriscina, M. (2012). Framing Inflation and Investment: The New York Times and the Cultural Context of a Local Housing Market. Qualitative Sociology, 35 (3), pp. 271-292. doi: https://doi.org/10.1007/s11133-012-9230-8

Lee, C. M, (1992). Earnings news and small traders. Journal of Accounting and Economics, 15, pp. 265-302. doi: https://doi.org/10.1016/0165-4101(92)90021-S

Lee, M. (2014). A Review of Communication Scholarship on the Financial Markets and the Financial Media. International Journal of Communication, 8, pp. 715-736.

Malo de Molina, J. L. (2014). ECB action and the Spanish economy during the first fifteen years of the euro. Bank of Spain Economic Bulletin, February, pp. 11-23.

McCollough, J. y Karani, K. (2014). The Media's Role in Influencing Perceptions of Housing Values and the Resulting Impact on the Macroeconomy. Economic Affairs, 34 (1), pp. 68-77. doi: https://doi.org/10.1111/ecaf.12047

Mercille, J. (2014). The Role of Media in Sustaining Ireland's Housing Bubble. New Political Economy, 19 (2), pp. 282-301. doi: https://doi.org/10.1080/13563467.2013.779652

Mercille, J. (2016). The Political Economy and Media Coverage of the European Economic Crisis: The case of Ireland. London: Routledge.

Müller, S. (2011). The Real State Bubble in Spain has been Pumped Up by All of Us. Aestimatio. The IEB International Journal of Finance, 2, pp. 2-11.

Neuendorf, K. A. (2002). The content analysis: Guide book. Thousand Oaks, CA: SAGE.

Ojala, J. y Uskali, T. (2007). Any weak signals?: the New York Times and the stock market 
crashes of 1929, 1987 and 2000. En Müller, L. y Ojala, J. (eds.). Information flows: new approaches in the historical study of business information (pp. 103-136). Helsinki: Suomalaisen kirjallisuuden seura..

Peress, J. (2014). The media and the diffusion of information in financial markets: evidence from newspaper strikes. The Journal of Finance, 69 (5), pp. 2007-2043. doi: https://doi. org/10.1111/jofi.12179

Peston, R. (2009). In the new digital world. There is a stronger need than ever for subsidised, public-service news. Ethical Space: The International Journal of Communication Ethics, 6 (3/4), pp. 10-21.

Picard, R. (ed.) (2015). The Euro Crisis in the Media. Journalistic Coverage of Economic Crisis and European Institutions. London: I.B Tauris \& The Reuters Institute for the Study of Journalism, University of Oxford.

Preston, P. y Silke, H. (2014). Ireland-From Neoliberal Champion to "the Eye of the Storm. Javnost-The Public, 21 (4), pp. 5-23. doi: https://doi.org/10.1080/13183222.2014.11077100 Quiring, O. y Weber, M. (2012). Between Usefulness and Legitimacy: Media Coverage of Governmental Intervention during the Financial Crisis and Selected Effects. The International Journal of Press/Politics, 17 (3), pp. 294-315. doi: https://doi. org/10.1177/1940161212442957

Roush, C. (2011). The Financial Press: It`s Not as Bad as Its Reputation. En Schiffrin, A. (ed.). Bad News. How America's Business Press Missed the Story of the Century (pp. 54-70). New York: The New Press.

Schifferes, S. y Roberts, R. (eds.) (2014). The Media and Financial Crises: Comparative and Historical Perspectives. London: Routledge.

Schiffrin, A. (ed.) (2011). Bad News. How America's Business Press Missed the Story of the Century. New York: The New Press.

Schuster, T. (2006). The Markets and the Media: Business News and Stock Market Movements. Lanham: Lexington Books.

Shiller, R. J. (2000). Irrational Exuberance. Princeton, NJ: Princeton University Press.

Shiller, R. J. (2008). The Subprime Solution. Princeton, NJ: Princeton University Press.

Shiller, R. J. (2001). Bubbles, Human Judgement, and Expert Opinion. Cowles Foundation Discussion Paper, № 1303 .

Silke, H. (2015). Base, Superstructure, and the Role of Journalism in the Irish Property Crash - Towards a Crisis Theory of Communication. tripleC, 13 (1), pp. 298-320.

doi: https://doi.org/10.31269/triplec.v13i2.660

Soo, C. K. (2015). Quantifying Animal Spirits: News Media and Sentiment in the Housing Market. Ross School of Business Working Papers, № 1200.

Starkman, D. (2014). The Watchdog That Didn't Bark: The Financial Crisis and the Disappearance of Investigative Journalism. New York: Columbia University Press.

Starr, M. A. (2012). Contributions of Economists to the Housing-Price Bubble. Journal of Economic Issues, 46 (1), pp. 143-172. doi: https://doi.org/10.2753/JEI0021-3624460106

Strauß, N., Vliegenthart, R. y Verhoeven, P. (2016) Lagging behind? Emotions in newspaper 
articles and stock market prices in the Netherlands. Public Relations Review, 42 (4), pp. 548-555.doi: https://doi.org/10.1016/j.pubrev.2016.03.010

Strauß, N., Vliegenthart, R. y Verhoeven, P. (2017) Intraday news trading: The reciprocal relationships between the stock market and economic news. Communication Research. Epub ahead of print 28 April. doi: https://doi.org/10.1177/0093650217705528.

Strauß, N. (2018). Financial journalism in today's high-frequency news and information era. Journalism. Epub ahead of print 28 January. doi: https://doi.org/10.1177/1464884917753556

Tetlock, P. C. (2007). Giving Content to Investor Sentiment: The Role of Media in the Stock Market. The Journal of Finance, 62 (3), pp. 1139-1168. doi: https://doi. org/10.1111/j.1540-6261.2007.01232.x

Theil, S. (2014). The Media and Markets: How Systematic Misreporting Inflates Bubbles, Deepens Downturns and Distorts Economic Reality. Discussion Paper 86, Shorenstein Center on Media, Politics and Public Policy, Harvard University.

Titley, G. (2012). Budgetjam! A communications intervention in the political-economic crisis in Ireland.Journalism, 14 (2), pp. 292-306. doi: https://doi.org/10.1177/1464884912448917

Tulloch, J. (2009). From amnesia to apocalypse: Reflections on journalism and the credit crunch. Ethical Space: The International Journal of Communication Ethics, 6 (3/4), pp. 99-109.

Uchitelle, L. (2011). The Uses and Misuses of Economics in Daily Journalism. History of Political Economy, 43 (2), pp. 363-368. doi: https://doi.org/10.1215/00182702-1257469

Usher, N. (2012). Ignored, uninterested, and the blame game: How the New York Times, Marketplace and The Street distanced themselves from preventing the 2007-2008 financial crisis. Journalism, 14 (2), pp. 190-207. doi: https://doi.org/10.1080/146167 0X.2015.1089183

Van Dalen, A., de Vreese, C. y Albæk, E. (2017). Economic News Through the Magnifying Glass. Journalism Studies, 18 (7), pp. 890-909. doi: https://doi.org/10.1080/146167 0X.2015.1089183

Walker, C. B. (2014) Housing booms and media coverage. Applied Economics, 46 (32), pp. 3954-3967. doi: https://doi.org/10.1080/00036846.2014.948675 